Check for updates

Cite this: RSC Adv., 2018, 8, 17410

\title{
Sensitive quantitative analysis of the bitter glycoside amarogentin by specific monoclonal antibody-based indirect competitive enzyme- linked immunosorbent assay $\dagger$
}

\begin{abstract}
Seiichi Sakamoto, D * Shinji Wada, Hiroyuki Tanaka* and Satoshi Morimoto
Amarogentin (AG) is a naturally occurring secoiridoid glycoside produced mainly in the plant genera Swertia and Gentiana. Extracts of these plants have a long history of use in Japan as bitter stomachics because of their strong bitterness. Because the AG content directly reflects the potential activity of the extract, the quality control of these plant extracts through the quantitative analysis of AG is important. In the present study, we aimed to develop a quantitative analysis of AG using a monoclonal antibody (MAb) against AG (MAb 1E9) in the plant family Gentianaceae. Surprisingly, production of MAb $1 E 9$ was successfully achieved by immunizing AG-bovine serum albumin (BSA) conjugates into mice although the number of $A G$ bound to BSA was only one. The characterization of MAb $1 E 9$ revealed that it shows high specificity to $A G$, enabling the development of an icELISA for the specific determination of AG. In addition, the detectable concentration of AG in the developed icELISA ranged from 1.95 to $62.5 \mathrm{ng} \mathrm{mL}^{-1}$ with a limit of detection of $1.28 \mathrm{ng} \mathrm{mL}^{-1}$, which is approximately 30-625 times higher in sensitivity compared with the conventional HPLC method. Validation analysis revealed that the icELISA using MAb 1 E9 is precise (intra-assay variation $<3.9 \%$, inter-assay variation $<8.8 \%$ ) and accurate (recovery rates of spiked samples were between $91.0 \%$ and $106.4 \%$ ). This method can be used for the quality control of plant extracts using $A G$ as an index.
\end{abstract}

Received 5th April 2018

Accepted 4th May 2018

DOI: $10.1039 / c 8 r a 02922 a$

rsc.li/rsc-advances

\section{Introduction}

Amarogentin [AG; Fig. 1(A)] is a naturally occurring secoiridoid glycoside produced mainly in plants belonging to the genera Swertia and Gentiana, both belonging to the family Gentianaceae. The extracts of members of this family have long been used as bitter stomachics because they contain bitter secoiridoid glycosides, including AG, amaroswerin (AS), swertiamarin, sweroside, and gentiopicroside (Fig. 1), all of which enhance gastrointestinal motility and the secretion of digestive juices. ${ }^{1-3}$ Among them, AG is known as the bitterest natural compound $^{4}$ and the root extract of Gentiana lutea is registered as a bitter food additive in the List of Existing Food Additives in Japan. ${ }^{5}$ In addition to the activity of AG as a bitter stomachic, AG has been shown to possess various pharmacological activities, including anti-diabetic activity, ${ }^{6}$ anti-leishmania activity,,${ }^{7,8}$ and hepatoprotective activities. ${ }^{9}$ More recently, AG was found to

Department of Pharmacognosy, Graduate School of Pharmaceutical Sciences, Kyushu University, 3-1-1 Maidashi, Higashi-ku, Fukuoka 812-8582, Japan. E-mail: s.sakamoto@phar.kyushu-u.ac.jp; htanaka@phar.kyushu-u.ac.jp; Fax: +8192642 6581; Tel: +81926426581

$\dagger$ Electronic supplementary information (ESI) available: The section for determination of hapten number and screening of test compounds for CRs test is available as ESI. See DOI: 10.1039/c8ra02922a induce the apoptosis of liver cancer cells by the upregulation of p53 and downregulation of human telomerase reverse transcriptase..$^{10}$ Thus, because the AG content directly reflects the potential activity of the extract, the quality control of Gentianaceae through the quantitative analysis of AG has recently attracted much attention. Currently, quantitative analysis for AG has been reported using liquid chromatography-mass spectrometry (LC-MS), ${ }^{11}$ high-performance liquid chromatography (HPLC), ${ }^{11,12}$ ultra-performance LC-electrospray ionization/MS, ${ }^{13}$ and capillary electrophoresis. ${ }^{14}$ However, these methods require large amounts of eco-unfriendly organic solvents, costly instruments, and time-consuming sample pretreatment.

In our previous studies, we focused on the quantitative analysis of plant secondary metabolites and developed various immunoassays, including enzyme-linked immunosorbent assays (ELISAs), fluorescence-linked immunosorbent assays, and immunochromatographic strip assays. These immunoassays exhibited several advantages over traditional analytical methods, such as HPLC, in terms of simplicity, selectivity, and sensitivity because the principle is based on a specific antigenantibody reaction. ${ }^{15}$

In the present study, we produced a specific monoclonal antibody (MAb) against AG (MAb 1E9) to develop an indirect 
(A)

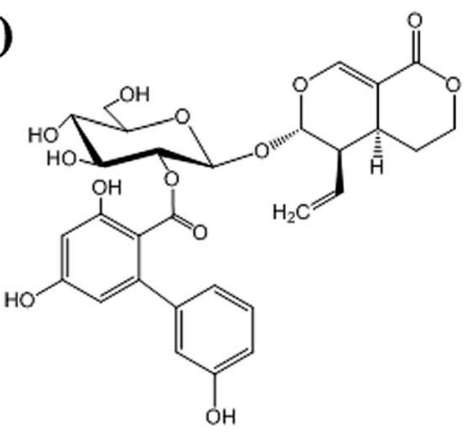

(B)

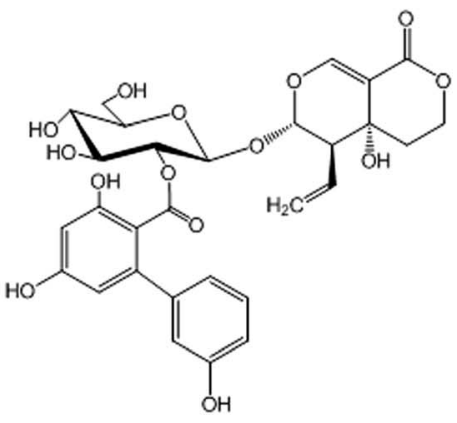

(C)

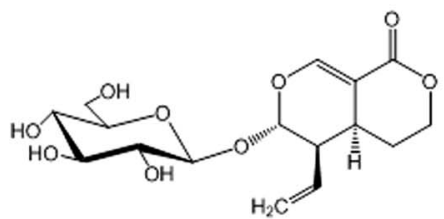

(D)

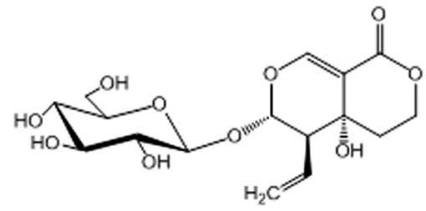

(E)

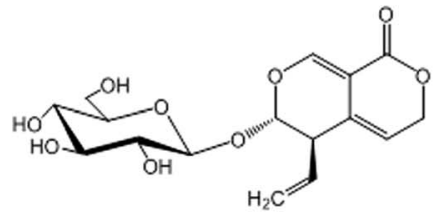

Fig. 1 Structures of major secoiridoid glycosides, amarogentin (AG; (A)), amaroswerin (AS; (B)), sweroside (C), swertiamarin (D), and gentiopicroside (E).

competitive ELISA (icELISA) for the determination of AG. AGbovine serum albumin (BSA) conjugates were prepared via $N, N^{\prime}$ carbonyldiimidazole (CDI) and were administered to BALB/c mice to induce an immunoresponse. Hybridomas secreting MAb 1E9 were obtained via the polyethylene glycol (PEG) method, and the MAb 1E9 were purified using affinity column chromatography. It is note that MAb 1E9 was successfully produced using AG-BSA conjugates possessing only one AG molecule per BSA molecule. The systematic characterization of MAb 1E9 revealed that it possesses high specificity to AG and high sensitivity, with a limit of detection (LOD) of $1.28 \mathrm{ng} \mathrm{mL}^{-1}$. To the best of our knowledge, this is now the most sensitive analytical method for determining the AG content in plant samples. Various validation analyses, including intra- and interassay tests, and spiked recovery tests alongside correlation analysis using HPLC support the accuracy and precision of the developed icELISA for the quality control of Gentianaceae through the quantitative analysis of AG.

This paper demonstrated the production of MAb 1E9 and its application to icELISA.

\section{Experimental section}

\section{Chemicals and reagents}

Amarogentin (AG, $\geq 95 \%$ ) was obtained from EXTRASYNTHESE (Lyon, France). Bovine serum albumin (BSA, $\geq 97 \%$ ), human serum albumin (HSA, $\geq 99 \%$ ), and ovalbumin (OVA, $\geq 98 \%$ ) were purchased from Sigma-Aldrich (St. Louis, MO, USA). Freund's complete and incomplete adjuvants were obtained from Difco (Detroit, MI, USA). Goat F(ab) Anti-Mouse IgG H\&L (HRP) (ab6823) was used as a secondary antibody and was purchased from Abcam (Cambridge, MA, USA). All the other chemicals were standard commercial products of analytical to reagent grade.

\section{Isolation of AS from Swertia japonica}

To evaluate the specificity of MAb 1E9 to AG, structurally similar compounds were required. Because AS [Fig. 1(B)] was commercially unavailable, it was isolated from $S$. japonica (powdered; Uchida Wakanyaku Co. Ltd., Tokyo, Japan). $S$. japonica $(\sim 200 \mathrm{~g})$ were soaked in methanol $(500 \mathrm{~mL})$, sonicated for $1 \mathrm{~h}$, and filtered. This extraction step was repeated five times, and the filtrates $(\sim 2.5 \mathrm{~L})$ were then concentrated under vacuum to obtain $\sim 53.5 \mathrm{~g}$ of extracts. The extracts were dissolved in $50 \%$ (v/v) $\mathrm{MeOH}$ and partitioned with hexane. The aqueous layer was then partitioned with $\mathrm{CHCl}_{3}$, followed by ethyl acetate, to obtain an ethyl acetate fraction ( $\sim 4.3 \mathrm{~g})$ containing AS. Reverse-phase chromatography using Cosmosil $75 \mathrm{C}_{18}$-OPN (Nacalai Tesque, Kyoto, Japan) with 50\% (v/v) MeOH and silica gel 60 (0.063$0.200 \mathrm{~mm}$; Merck, Darmstadt, Germany) chromatography with a mixture of $\mathrm{CHCl}_{3}$ and $\mathrm{MeOH}\left(\mathrm{CHCl}_{3} / \mathrm{MeOH}=8 / 2\right)$ was performed to obtain AS ( $210 \mathrm{mg})$. NMR spectra were recorded on a Varian Unity 500 plus apparatus (Varian, Palo Alto, CA, USA) operating at $500 \mathrm{MHz}$ for ${ }^{1} \mathrm{H}$ NMR and $125 \mathrm{MHz}$ for ${ }^{13} \mathrm{C}$ NMR in methanol- $d_{4}$.

\section{Animals}

BALB/c mice (5 weeks old, male) were purchased from KBT Oriental Co., Ltd. (Saga, Japan). A standard diet (MF; Oriental Yeast Co., Tokyo, Japan) and water were provided ad libitum. The animal experiment conducted in the present study was 
approved by the Committee on the Ethics of Animal Experiments, Kyushu University (approval no.: A30-003-0), and the experimental procedures were performed in accordance with the Guidelines for Animal Experiments of the Graduate School of Pharmaceutical Sciences, Kyushu University.

\section{Preparation of plant samples}

Seven kinds of Gentianaceae were analyzed using icELISA and HPLC. S. japonica (powdered), S. japonica (whole plant), G. lutea (powdered), and G. scabra (powdered) were commercially obtained from Uchida Wakanyaku Co. Ltd., (Tokyo, Japan). S. japonica (whole plant, Nagano, Japan), G. scabra I (root), and G. scabra II (root; Ibaraki, Japan) were obtained from voucher specimens deposited at the Herbarium of the Faculty of Pharmaceutical Sciences, Kyushu University. Powdered samples were measured without any treatment, whereas whole plants and roots were ground into a fine powder and sieved through a $300 \mu \mathrm{m}$ mesh filter. Plant powder $(50 \mathrm{mg})$ was measured and immersed in $\mathrm{MeOH}(1 \mathrm{~mL})$. Extraction was performed by sonication for $20 \mathrm{~min}$, and the supernatant, after centrifugation at $10000 \mathrm{rpm}$ for $10 \mathrm{~min}$, was collected in small test tubes. This step was repeated three times. The combined $\mathrm{MeOH}$ extract (3 $\mathrm{mL}$ ) was concentrated to dryness under flowing nitrogen. The residue was then dissolved in $\mathrm{MeOH}(1.0 \mathrm{~mL})$ and used as a sample for both icELISA and HPLC.

\section{Preparation of AG-BSA, AG-HSA, and AG-OVA conjugates}

AG possesses a hydroxyl group; therefore, AG was conjugated to proteins (BSA, HSA, and OVA) via CDI, which is a zero-length cross-linking reagent. ${ }^{16}$ For the AG-BSA conjugates used as immunogens, AG $(3.1 \mathrm{mg})$ and CDI $(3.0 \mathrm{mg})$ were dissolved in dimethylformamide $(0.6 \mathrm{~mL})$, which was stirred at $30^{\circ} \mathrm{C}$ for $3 \mathrm{~h}$. Subsequently, this solution was added dropwise to another solution, where BSA ( $4.2 \mathrm{mg}$ ) was dissolved in distilled water $(2.7 \mathrm{~mL})$. The resulting mixture was then stirred at $30{ }^{\circ} \mathrm{C}$ for $20 \mathrm{~h}$. The reaction mixture was dialyzed five times against distilled water at $4{ }^{\circ} \mathrm{C}$ for 2 days and then lyophilized to obtain AG-BSA conjugates (3.9 mg). AG-HSA (3.8 mg) and AG-OVA (3.6 $\mathrm{mg}$ ) conjugates were prepared in the same manner using HSA (3.9 mg) and OVA (4.1 mg), respectively. All conjugates were dissolved in $50 \mathrm{mM}$ Tris- $\mathrm{HCl}(\mathrm{pH} 8.0)$ containing $8 \mathrm{M}$ urea at $20 \mathrm{mg} \mathrm{mL}^{-1}$ and stored at $-20{ }^{\circ} \mathrm{C}$ until use.

\section{Production of MAb 1E9}

Immunization of BALB/c mice ( 5 weeks old, male) was performed with AG-BSA conjugates every 2 weeks, as described previously. ${ }^{17}$ At the first and second immunizations, Freund's complete and incomplete adjuvants were used to enhance the immune response by forming an emulsion with the immunogen (AG-BSA conjugates). The resulting emulsions, corresponding to $50 \mu \mathrm{g}$ of AG-BSA conjugates, were administered intraperitoneally. Booster shots were administered three times by immunization with AG-BSA conjugates $(100 \mu \mathrm{g})$ without using adjuvants. On the fifth day after the final booster, the splenocytes were prepared and fused with SP2/0 mice myeloma cells using the PEG method. The fused cells were then cultured in a selection medium containing enriched RPMI 1640-Dulbecco's-Ham's F12 (eRDF; Kyokuto Pharmaceutical Industrial Co., Tokyo, Japan) medium supplemented with hypoxanthineaminopterin-thymidine (HAT), RD-1 additives (Kyokuto Pharmaceutical Industrial Co., Tokyo, Japan), and $10 \%(\mathrm{v} / \mathrm{v})$ fetal calf serum (FCS; Gibco-Invitrogen, Carlsbad, CA, USA) at $37{ }^{\circ} \mathrm{C}$ in a humidified incubator and a $5 \% \mathrm{CO}_{2}$ atmosphere. Hybridomas secreting anti-AG antibody were then cloned by the limited dilution method and selected by direct ELISA and icELISA. The selected hybridomas (1E9) were scaled up in the same buffer without HAT until the volume became $1 \mathrm{~L}$. Finally, the medium was exchanged to a serum-free medium (without FCS) and further cultured for 1 week.

Purification of MAb 1E9 was performed using affinity column chromatography. Briefly, the culture medium containing MAb 1E9 was centrifuged, and the $\mathrm{pH}$ of the supernatant was adjusted to $\mathrm{pH} 7.0$ with $1 \mathrm{M}$ Tris- $\mathrm{HCl}$ buffer ( $\mathrm{pH} 9.0)$. The culture medium was then filtered, and the filtrates were subjected to chromatography on a Protein G Sepharose 4 Fast Flow (GE Healthcare Bio-Science AB, Uppsala, Sweden) column equilibrated with $10 \mathrm{mM}$ phosphate buffer $(\mathrm{pH}$ 7.0). The bound proteins were then washed with $10 \mathrm{mM}$ phosphate buffer $(\mathrm{pH}$ 7.0) and eluted with $100 \mathrm{mM}$ citrate buffer ( $\mathrm{pH}$ 2.7). Ten test tube fractions containing MAb 1E9 were obtained. To avoid inactivation of the eluted MAb 1E9, the eluates $(4.0 \mathrm{~mL})$ were immediately neutralized with $1 \mathrm{M}$ Tris-HCl buffer $(\mathrm{pH} 9.0 ; 1.4$ $\mathrm{mL}$ ). Subsequently, all fractions were collected, concentrated, dialyzed against distilled water at $4{ }^{\circ} \mathrm{C}$ for 1 week, and lyophilized to yield $79.0 \mathrm{mg}$ of MAb $1 \mathrm{E} 9$.

\section{Indirect ELISA and icELISA using MAb 1E9}

The binding reactivity of MAb 1E9 was evaluated by ELISA using AG-HSA as the coated antigen. The reactivity against AG-HSA conjugates and free AG molecules was investigated by indirect ELISA and icELISA, respectively. In both ELISA tests, AG-HSA conjugates $\left(5 \mu \mathrm{g} \mathrm{mL}^{-1}\right)$ in $50 \mathrm{mM}$ carbonate buffer ( $\mathrm{pH} 9.6 ; 100$ $\mu \mathrm{L}$ per well) were immobilized to the surface of a 96-well immunoplate (Nunc, Maxisorb, Roskilde, Denmark) by incubation at $37{ }^{\circ} \mathrm{C}$ for $1 \mathrm{~h}$. After washing the plate, the wells were then treated with PBS containing 5\% (w/v) skimmed milk (300 $\mu \mathrm{L}$ per well) at $37{ }^{\circ} \mathrm{C}$ for $1 \mathrm{~h}$ to block and reduce non-specific adsorption of other proteins. The following step, using MAb 1E9, was different between indirect ELISA and icELISA. For indirect ELISA, various concentrations of MAb $1 \mathrm{E} 9(100 \mu \mathrm{L}$ per well) in PBS containing $0.05 \%(\mathrm{v} / \mathrm{v})$ Tween 20 (PBS-T) were allowed to react with the coated AG-HSA conjugates. For icELISA, free AG molecules ( $50 \mu \mathrm{L}$ per well) in $5 \%(\mathrm{v} / \mathrm{v}) \mathrm{MeOH}$ were mixed with MAb $1 \mathrm{E} 9$ ( $50 \mu \mathrm{L}$ per well; $900 \mathrm{ng} \mathrm{mL}^{-1}$ ) in PBS-T, leading to a competitive reaction between free AG molecules and AG of coated AG-HSA conjugates against MAb 1E9. After incubation at $37^{\circ} \mathrm{C}$ for $1 \mathrm{~h}$ and a subsequent washing step, MAb 1E9 binding to the immobilized AG-HSA conjugates was reacted with Goat F(ab) Anti-Mouse IgG H\&L (HRP) (100 $\mu \mathrm{L}$ per well), which was 5000 times diluted with PBS-T, at $37^{\circ} \mathrm{C}$ for $1 \mathrm{~h}$. Finally, the $0.3 \mathrm{mg} \mathrm{mL}{ }^{-1} 2,2^{\prime}$-azino-bis(3-ethylbenzothiazoline6-sulfonic acid)diammonium salt substrate solution in 
$100 \mathrm{mM}$ citrate buffer ( $\mathrm{pH} 4.0$ ) supplemented with $0.003 \%(\mathrm{v} / \mathrm{v})$ $\mathrm{H}_{2} \mathrm{O}_{2}\left(100 \mu \mathrm{L}\right.$ per well) was added and incubated at $37^{\circ} \mathrm{C}$ for $15 \mathrm{~min}$ to develop color.

All the washing steps were performed thrice using PBS-T between each step. The developed color was detected based on the absorbance at $405 \mathrm{~nm}$ using a microplate reader (Multiskan $^{\mathrm{TM}}$ FC microplate photometer, Thermo Fisher Scientific, Inc., Waltham, MA, USA). The cross-reactivities (CRs) of MAb 1E9 were evaluated using the formula described by Weiler and Zenk as follows: ${ }^{18}$

$$
\mathrm{CRs}(\%)=\frac{\mathrm{IC}_{50} \text { for } \mathrm{AG}}{\mathrm{IC}_{50} \text { for compound under investigation }} \times 100
$$

\section{Recovery of spiked AG}

Various amounts of AG $(7.5,15$, and $30 \mu \mathrm{g})$ were spiked into $\mathrm{MeOH}$ solution ( $1 \mathrm{~mL}$ ) extracted from $S$. japonica (powdered). In the unspiked samples, $95.86 \mu \mathrm{g}$ of AG was found per $50 \mathrm{mg}$ of dried sample by icELISA. After spiking with AG standards, the amounts of AG were determined using the developed icELISA and the recovery rate was calculated as follows:

$$
\begin{aligned}
\text { Recovery rate }(\%)= & \frac{\text { measured amount of } \mathrm{AG}-95.86 \mu \mathrm{g}}{\text { spiked amount of } \mathrm{AG}} \\
& \times 100
\end{aligned}
$$

\section{HPLC analysis}

HPLC analysis was performed according to the method by Amakura et al., with slight modification. ${ }^{12}$ An LC-10AD VP HPLC pump connected to a SPD-10A UV/VIS detector $(254 \mathrm{~nm})$ and CR8A chromatopac, all of which were Shimadzu products (Kyoto, Japan), were used in the analysis. After the samples $(20 \mu \mathrm{L})$ were subjected to a reversed-phase column (Cosmosil-packed $5 \mathrm{C}_{18}$ AR II $4.6 \mathrm{~mm} \times 150 \mathrm{~mm}, 5 \mu \mathrm{m}$ particle size, Nacalai Tesque, Kyoto, Japan), equilibrated with a mobile phase comprising $20 \%(\mathrm{v} / \mathrm{v})$ acetonitrile with $0.5 \%(\mathrm{v} / \mathrm{v})$ acetic acid solution, the eluates were analyzed for $20 \mathrm{~min}$. AG was detected at the retention time of $\sim 17.0 \mathrm{~min}$ when the flow rate was 1.0 $\mathrm{mL} \min ^{-1}$. The calibration curve for AG was drawn in the concentration range of $0.049-12.5 \mu \mathrm{g} \mathrm{mL}^{-1}$ with a LOD of 0.23 $\mu \mathrm{g} \mathrm{mL}^{-1}$. All sample analyses were performed in triplicate.

\section{Results and discussion}

\section{Determination of hapten numbers by MALDI-TOF-MS}

The number of AG molecules bound to the BSA carrier protein was evaluated by MALDI-TOF-MS analysis (Bruker Autoflex III). In this analysis, the BSA and AG-BSA conjugates appeared at $\mathrm{m} / \mathrm{z}$ 66451 and $m / z 67224$, respectively (Fig. S1†). By taking the molecular weight of AG (586.54) into account, at least one molecule of AG was found to be conjugated to a BSA molecule. Approximately 40 years ago, the appropriate hapten numbers had already been investigated by Erlanger for the production of antihapten antibodies using BSA conjugates and he concluded that between 8 and 25 molecules were optimal. ${ }^{19}$ However, in our recent reports, highly specific and highly sensitive antibodies

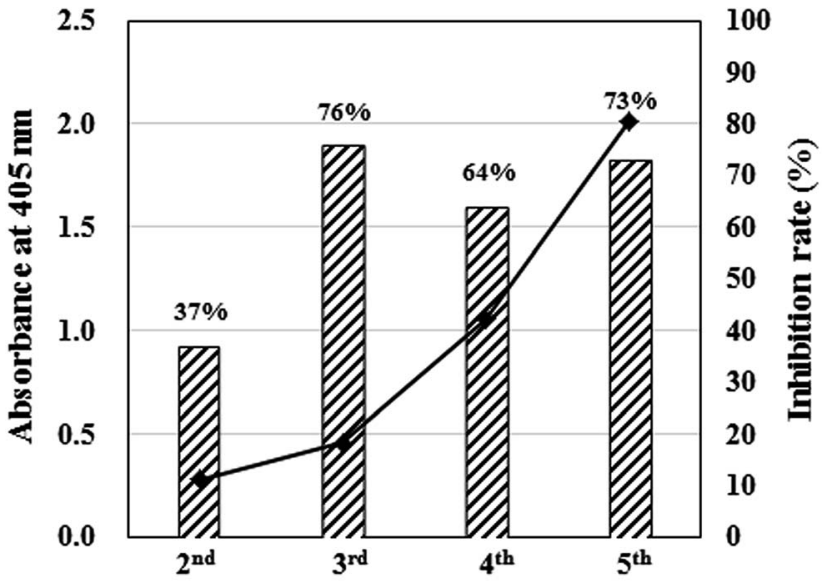

Immunization times of AG-BSA conjugates

Fig. 2 Antibody titer and its inhibition rate against AG evaluated by ELISAs. The closed squares and hatched bar graphs indicate absorbance at $405 \mathrm{~nm}$ evaluated by indirect ELISA and inhibition rate against AG $\left(50 \mu \mathrm{g} \mathrm{mL}^{-1}\right)$. In both ELISAs, AG-OVA conjugates $\left(1 \mu \mathrm{g} \mathrm{mL}^{-1}\right)$ were used as coated antigen, and 100 times diluted antisera with PBS-T were used as primary antibody.

were successfully produced against harringtonine, ${ }^{\mathbf{2 0}}$ homoharringtonine, $^{\mathbf{2 1}}$ and monocrotaline, ${ }^{22}$ despite only having hapten numbers of 2 per BSA molecule. Thus, AG-BSA conjugates obtained herein were used as immunogens for further antibody production.

\section{Production and typing of MAb 1E9}

On the fourth day after immunization, mouse serum was obtained from the tail vein of $\mathrm{BALB} / \mathrm{c}$ mice, and the titer and inhibitory activity against $\mathrm{AG}\left(50 \mu \mathrm{g} \mathrm{mL} \mathrm{m}^{-1}\right)$ in the serum were investigated by indirect ELISA and icELISA, respectively (Fig. 2). Interestingly, despite the hapten number of 1 , the antibody titer increased as the immunization times increased. In addition, icELISA revealed that the antibody in the serum obviously inhibits free AG. Since the antibody titer $\left(\mathrm{OD}_{405}=2.01\right)$ and inhibition rate against AG $(73 \%)$ increased enough to be used in ELISA after the fifth immunization (Fig. 2), cell fusion between splenocytes and SP2/0 mice myeloma cells was performed using the PEG method. After several screenings, only one hybridomas (1E9) secreting anti-AG antibody was obtained and scaled up to purify MAb $1 \mathrm{E} 9$.

The isotype of MAb 1E9 was identified using an IsoStrip Mouse Monoclonal Antibody Isotyping Kit (Roche Diagnostics, Mannheim, Germany), and this revealed that the MAb 1E9 was an IgG1 that possessed a $\kappa$ light chain.

\section{Optimization of icELISA using MAb 1E9}

The binding reactivity of MAb $1 \mathrm{E} 9$ against AG-HSA conjugates $\left(5 \mu \mathrm{g} \mathrm{mL}{ }^{-1}\right)$ was analyzed by indirect ELISA. The absorbance against $\log [\mathrm{MAb} 1 \mathrm{E} 9]$ was plotted for the reactivity response curve. The concentration of MAb 1E9 positively correlated with the absorbance in a logarithmic manner [Fig. 3(A)]. This result 
(A)

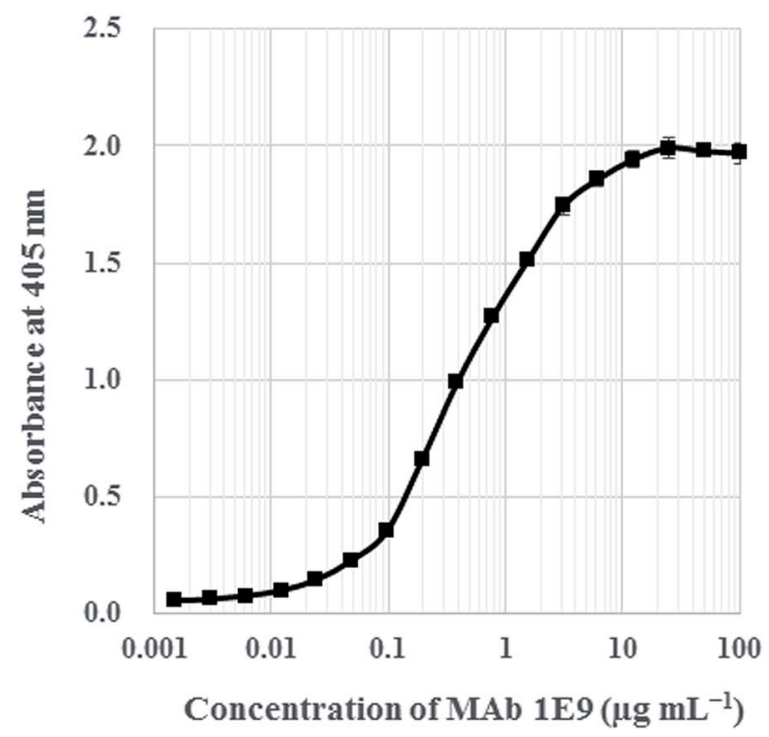

(B)

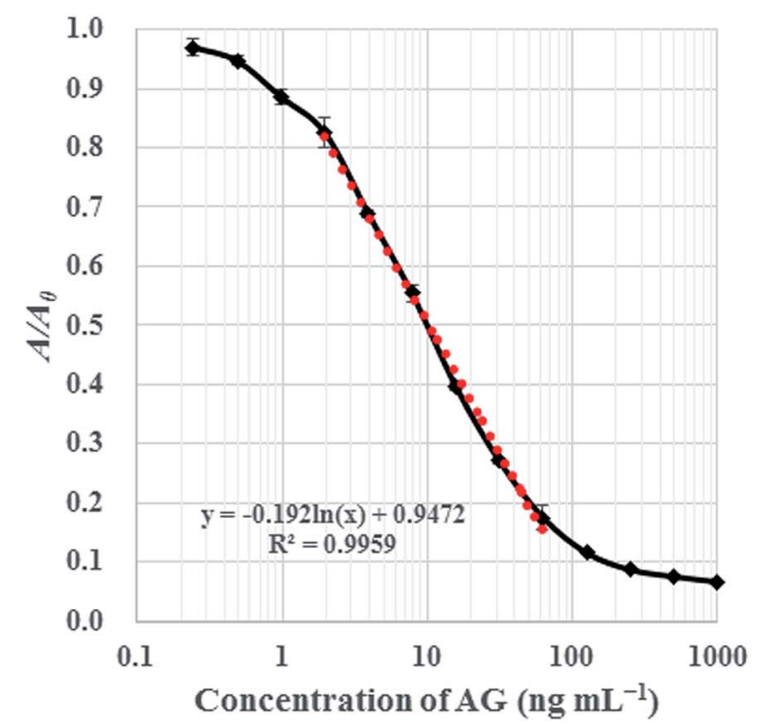

Fig. 3 Reactivity response curves in indirect ELISA against AG-HSA conjugates (A) and icELISA against free AG (B). In both ELISAs, concentration of AG-HSA conjugates was $5 \mu \mathrm{g} \mathrm{mL} \mathrm{L}^{-1}$. In icELISA (B), $A_{0}$ and $A$ corresponds the absorbance in the absence and presence of AG. A dotted line represents linearized curve for $A G$ with the determination range from 1.95 to $62.5 \mathrm{ng} \mathrm{mL}^{-1}$.

Table 1 Comparison of LOD between developed icELISA and reported HPLC system

\begin{tabular}{ll}
\hline Method & LOD \\
\hline icELISA & $1.28 \mathrm{ng} \mathrm{mL}^{-1}$ \\
HPLC & $37 \mathrm{ng} \mathrm{mL}^{-1}$ (ref. 11) \\
& $0.8 \mu \mathrm{gL} \mathrm{m}^{-1}$ (ref. 12)
\end{tabular}

revealed that $450 \mathrm{ng} \mathrm{mL}^{-1}$ of MAb $1 \mathrm{E} 9$ gives an absorbance of $\sim 1.0$ in the indirect ELISA test, suggesting that $900 \mathrm{ng} \mathrm{mL}^{-1}$ is optimal for MAb 1E9 as a primary antibody in icELISA because the volume of primary antibody in icELISA $(50 \mu \mathrm{L})$ should be half of that in an indirect ELISA $(100 \mu \mathrm{L})$, as mentioned above.

Then, the inhibitory activity of MAb 1E9 against free AG was analyzed by icELISA. The AG standard was serially diluted with $5 \%(\mathrm{v} / \mathrm{v}) \mathrm{MeOH}$, and applied (50 $\mathrm{LL}$ per well) to the immunoplate coated with AG-HSA conjugates. MAb 1E9 diluted with PBS-T (900 ng mL ${ }^{-1} ; 50 \mu \mathrm{L}$ well ${ }^{-1}$ ) was then added and incubated to allow for competition between free AG/AG-HSA conjugates and MAb 1E9. The increased free AG led to a decrease in the amount of MAb 1E9 that could bind to AGHSA conjugates, and vice versa; thus, the absorbance decreased as the MAb 1E9 concentration increased in a logarithmic manner. In this icELISA, the detectable concentration of AG was in the range of $1.95-62.5 \mathrm{ng} \mathrm{mL}^{-1}$ with a LOD of $1.28 \mathrm{ng} \mathrm{mL}^{-1}$ [Fig. 3(B)]. When the sensitivity of the developed icELISA was compared with that of HPLC systems on the basis of LOD, it was found that this icELISA test is $\sim 180$ times more sensitive than our developed HPLC system (LOD $=0.24 \mu \mathrm{g} \mathrm{mL} \mathrm{m}^{-1}$ ) and $\sim 30$ -
625 times more sensitive than previously reported HPLC systems (Table 1).

\section{CR test of MAb 1E9}

Specificity and sensitivity are two of the most important factors in quantitative analysis. Specificity in ELISA can be evaluated from cross-reaction of antibodies. To investigate the specificity of MAb 1E9 against AG, structurally similar compounds to AG were necessary. Because AS [Fig. 1(B)] was commercially unavailable, it was isolated from powdered $S$. japonica and identified as AS by comparison with reported ${ }^{1} \mathrm{H}$ and ${ }^{13} \mathrm{C}$ NMR spectroscopic data. ${ }^{23}$ For screening, the first icELISA was performed using 41 kinds of natural products at a concentration of $50 \mu \mathrm{g} \mathrm{mL} L^{-1}$ (Fig. $\mathrm{S} 2 \dagger$ ). This screening revealed that MAb 1E9 shows no recognition against 37 kinds of compounds that do not structurally resemble AG. In contrast, MAb 1E9 exhibited clear inhibition against four secoiridoid glycosides, which include AS, swertiamarin, sweroside, and gentiopicroside (Fig. 1); therefore, these four secoiridoid glycosides were serially diluted with $5 \%(\mathrm{v} / \mathrm{v}) \mathrm{MeOH}$ and applied to a second icELISA for

Table 2 Cross-reactivity (CR) of MAb $1 E 9$ against secoiridoid glycosides

\begin{tabular}{llr}
\hline Class & Compound & CR (\%) \\
\hline Secoiridoid glycosides & Amarogentin (AG) & 100.00 \\
& Amaroswerin (AS) & 2.28 \\
& Sweroside & 0.84 \\
& Swertiamarin & 0.04 \\
& Gentiopicroside & 0.04
\end{tabular}


Table 3 Intra- and inter-assay precision tests of icELISA using MAb $1 \mathrm{E} 9^{a}$

\begin{tabular}{lll}
\hline & \multicolumn{2}{c}{$(\%)$} \\
\cline { 2 - 3 } $\begin{array}{ll}\text { Concentration } \\
\text { of AG }\left(\mathrm{ng} \mathrm{mL}^{-1}\right)\end{array}$ & $\begin{array}{l}\text { Intra-assay } \\
(n=6)\end{array}$ & $\begin{array}{l}\text { Inter-assay } \\
(n=5)\end{array}$ \\
\hline 62.5 & 3.86 & 8.75 \\
31.3 & 3.07 & 6.48 \\
15.6 & 1.28 & 4.39 \\
7.81 & 1.87 & 3.60 \\
3.91 & 1.59 & 3.82 \\
1.95 & 1.50 & 2.88
\end{tabular}

${ }^{a}$ All values represent coefficient of variation (CV) calculated from formula as follow: $\mathrm{CV}=$ standard deviation (S.D.)/mean $\times 100$.

further CR testing. Their CRs were calculated from the ratio of the $\mathrm{IC}_{50}$ of $\mathrm{AG}$ to that of the four test compounds. CR testing revealed that MAb 1E9 possesses high specificity to AG because the CRs against the four secoiridoid glycoside test compounds were $<3 \%$ (Table 2). The structural difference between AG and sweroside is the trihydroxy-biphenyl carboxylic acid moiety. Considering the drastic decrease in CR against sweroside (CRs: $0.84 \%$ ), it can be stated that the trihydroxy-biphenyl carboxylic acid moiety plays an important role in conformational recognition by MAb 1E9. Moreover, the recognition by MAb 1E9 against AG (CRs: 100\%) was found to be different from that against AS (CRs: 2.28\%), although the structural difference between AG and AS is only the hydroxyl group at the C5 position; this suggests that this functional group contributes greatly to
MAb 1E9 recognition as well as the trihydroxy-biphenyl carboxylic acid moiety. Collectively, MAb 1E9 specifically recognizes the entire AG molecule.

This highly specific recognition of AG by MAb 1E9 suggests that it can be applied to the specific determination of AG using icELISA.

\section{Validation analysis of icELISA using MAb 1E9}

The precision of the icELISA using MAb 1E9 was investigated using intra- and inter-assay tests, while its accuracy was examined via a recovery test.

The intra-assay precision was evaluated by observing the $405 \mathrm{~nm}$ absorbance variation from well to well in the same plate $(n=6)$. The inter-assay precision was evaluated by observing the variations from different plates $(n=5)$ at six AG concentrations within the detectable range $(62.5,31.3,15.6,7.81,3.91$, and 1.95 $\mathrm{ng} \mathrm{mL}^{-1}$ ). The maximum coefficient of variation in the intraassay test was $3.86 \%$ whereas that in the inter-assay test was $8.75 \%$, indicating that the developed icELISA possesses high repeatability for the determination of AG (Table 3).

The accuracy of icELISA was further analyzed via the recovery test. In the recovery test, various amounts of $\operatorname{AG}(7.5,15$, and 30 $\mu \mathrm{g})$ were spiked into $\mathrm{MeOH}$ extracts obtained from powdered $S$. japonica and the recovery rate was calculated using the measured amount of AG by icELISA and the spiked amount (Table 4). These results show that the most spiked AG sample is recovered within rates between $91.0 \%$ and $106.4 \%$, supporting the accuracy of the developed icELISA and the usefulness of MAb 1E9 as a tool for reliable AG determination in plant samples.

Table 4 Recovery of spiked AG from $\mathrm{MeOH}$ extract of $S$. japonica determined by icELISA

\begin{tabular}{llll}
\hline $\begin{array}{l}\text { Spiked amount } \\
\text { of AG }(\mu \mathrm{g})\end{array}$ & $\begin{array}{l}\text { Measured amount of } \mathrm{AG}^{a}(\mu \mathrm{g} \\
\text { per } 50 \mathrm{mg} \text { dry wt) }\end{array}$ & $\mathrm{CV}^{b}(\%)$ & $\begin{array}{l}\text { Expected amount } \\
\text { of AG }(\mu \mathrm{g} \text { per } 50 \mathrm{mg} \text { dry wt })\end{array}$ \\
\hline 0 & $95.86 \pm 8.56$ & 8.93 & \\
7.5 & $102.68 \pm 1.36$ & 1.32 & 103.36 \\
15 & $111.82 \pm 8.83$ & 7.90 & 110.86 \\
30 & $127.61 \pm 1.51$ & 1.19 & 125.86
\end{tabular}

${ }^{a}$ All values were mean \pm standard deviation (S.D.) from triplicate samples for each level. ${ }^{b}$ Coefficient of variation $($ CV $)=$ S.D. $/$ mean $\times 100$.

${ }^{c}$ Recovery $(\%)=($ measured amount $-95.86 \mu \mathrm{g}$ per spiked mount $) \times 100$.

Table 5 Determination of AG in the members of plant family Gentianaceae by developed icELISA and HPLC ${ }^{a}$

\begin{tabular}{|c|c|c|c|c|}
\hline \multirow[b]{2}{*}{ Sample name } & \multicolumn{2}{|l|}{ icELISA } & \multicolumn{2}{|l|}{ HPLC } \\
\hline & $\%$ (wt/dry wt) & CV $(\%)$ & $\begin{array}{l}\% \\
\text { (wt/dry wt) }\end{array}$ & $\mathrm{CV}(\%)$ \\
\hline S. japonica (powdered) & $1.26 \times 10^{-1} \pm 6.96 \times 10^{-3}$ & 5.51 & $0.886 \times 10^{-1} \pm 2.26 \times 10^{-3}$ & 2.55 \\
\hline S. japonica (whole plant) & $1.95 \times 10^{-1} \pm 7.63 \times 10^{-3}$ & 3.91 & $1.33 \times 10^{-1} \pm 3.58 \times 10^{-3}$ & 2.70 \\
\hline S. japonica (whole plant, Nagano, Japan) & $1.17 \times 10^{-1} \pm 7.11 \times 10^{-3}$ & 6.07 & $0.811 \times 10^{-1} \pm 0.963 \times 10^{-3}$ & 1.19 \\
\hline G. lutea (powdered) & $3.91 \times 10^{-2} \pm 2.38 \times 10^{-3}$ & 6.10 & Not quantitated & \\
\hline G. scabra (powdered) & $2.61 \times 10^{-3} \pm 5.08 \times 10^{-5}$ & 1.94 & Not detected (ND) & \\
\hline G. scabra I (root) & $9.45 \times 10^{-4} \pm 7.53 \times 10^{-5}$ & 7.97 & ND & \\
\hline G. scabra II (root, Ibaraki, Japan) & $3.80 \times 10^{-3} \pm 1.19 \times 10^{-4}$ & 3.13 & ND & \\
\hline
\end{tabular}


Determination of AG in members of the plant family Gentianaceae by icELISA and HPLC

The AG content in members of the plant family Gentianaceae $(S$. japonica, G. lutea, and G. scabra) was determined using the newly developed icELISA method and HPLC (Table 5). The AG content in $S$. japonica, as determined by icELISA, was positively correlated with that determined by HPLC. Regarding G. lutea, AG was not determined by HPLC because the AG content was below LOD $\left(0.24 \mu \mathrm{g} \mathrm{mL} \mathrm{m}^{-1}\right)$; however, the high sensitivity of icELISA enabled AG detection. With respect to G. scabra, small amounts of AG were detected by icELISA but no AG was detected by HPLC. To date, there have been no reports regarding the presence of AG in G. scabra; however, G. scabra has been reported to contain much gentiopicroside and sweroside (1-3\% wt/dry wt). ${ }^{24}$ Note that gentiopicroside and sweroside had slight CRs to MAb 1E9 (Table 2). These slight CRs, in contrast to the significant amounts of gentiopicroside and sweroside in $G$. scabra may result in a false positive for the detection of AG by icELISA. However, the presence of AG in G. scabra needs to be investigated further.

\section{Conclusions}

In the present study, MAb 1E9, which is highly specific to AG, was successfully prepared using AG-BSA conjugates despite only one AG being bound to BSA. Considering our recent reports about specific MAbs prepared using BSA conjugates containing only two target molecules, ${ }^{\mathbf{2 0 - 2 2}}$ very low hapten numbers may be sufficient for producing highly specific antibodies although some study concluded between 8 and 25 hapten number were optimal. ${ }^{19,25}$ A sensitive icELISA using MAb 1E9 was developed with a LOD of $1.28 \mathrm{ng} \mathrm{mL} \mathrm{m}^{-1}$, enabling the determination of small amounts of AG in members of the plant family Gentianaceae without the need for complicated pretreatment, which is commonly required in other analytical methods. MAb 1E9, which is specific to AG, could be useful for the quality control of Gentianaceae through quantitative AG analysis using the newly developed icELISA.

\section{Conflicts of interest}

The authors declare no competing financial interest.

\section{References}

1 B. Dutt, L. J. Srivastava and J. M. Singh, Anc. Sci. Life, 2009, 15, 226-229.

2 F. Mirzaee, A. Hosseini, H. B. Jouybari, A. Davoodi and M. Azadbakht, J. Tradit. Complement. Med., 2017, 7, 400-408.
3 Y. Niiho, T. Yamazaki, Y. Nakajima, T. Yamamoto, H. Ando, Y. Hirai, K. Toriizuka and Y. Ida, J. Nat. Med., 2006, 60, 82-88.

4 S. Rodriguez, A. Marston, J. L. Wolfender and K. Hostettmann, Curr. Org. Chem., 1998, 2, 627-648.

5 The Ministry of Health, Labour and Welfare of Japan, Notification No. 120, Apr. 16, 1996.

6 H. S. Niu, P. C. Chao, P. M. Ku, C. S. Niu, K. S. Lee and J. T. Cheng, Naunyn-Schmiedeberg's Arch. Pharmacol., 2016, 389, 1215-1223.

7 S. Medda, S. Mukhopadhyay and M. K. Basu, J. Antimicrob. Chemother., 1999, 44, 791-794.

8 S. Ray, H. K. Majumder, A. K. Chakravarty, S. Mukhopadhyay, R. R. Gil and G. A. Cordell, J. Nat. Prod., 1996, 59, 27-29.

9 Y. Zhang, H. Zhao, H. Li, W. Cao, F. Wang, T. Zhang and S. W. Wang, Molecules, 2017, 22, 754.

10 C. Huang, R. Li, Y. Zhang and J. Gong, Technol. Cancer Res. Treat., 2017, 16, 546-558.

11 A. Aberham, S. Schwaiger, H. Stuppner and M. Ganzera, J. Pharm. Biomed. Anal., 2007, 45, 437-442.

12 Y. Amakura, M. Yoshimura, S. Morimoto, T. Yoshida, A. Tada, Y. Ito, T. Yamazaki, N. Sugimoto and H. Akiyama, Chem. Pharm. Bull., 2016, 64, 78-82.

13 V. Kumar and S. Chandra, Physiol. Mol. Biol. Plants, 2015, 21, 51-60.

14 I. Citová, M. Ganzera, H. Stuppner and P. Solich, J. Sep. Sci., 2008, 31, 195-200.

15 S. Sakamoto, W. Putalun, S. Vimolmangkang, W. Phoolcharoen, Y. Shoyama, H. Tanaka and S. Morimoto, J. Nat. Med., 2018, 72, 32-42.

16 R. Paul and G. W. Anderson, J. Am. Chem. Soc., 1960, 82, 4596-4600.

17 S. Sakamoto, G. Yusakul, B. Pongkitwitoon, M. K. Paudel, H. Tanaka and S. Morimoto, Food Chem., 2015, 169, 127-133.

18 E. W. Weiler and M. H. Zenk, Phytochemistry, 1976, 15, 15371545.

19 B. F. Erlanger, Methods Enzymol., 1980, 70, 85-104.

20 S. Sakamoto, G. Yusakul, Y. Tsuneura, W. Putalun, K. Usui, T. Miyamoto, H. Tanaka and S. Morimoto, Analyst, 2017, 142, 1140-1148.

21 B. Pongkitwitoon, S. Sakamoto, R. Nagamitsu, W. Putalun, H. Tanaka and S. Morimoto, Planta Med., 2018, DOI: 10.1055/a-0578-8689.

22 S. Sakamoto, R. Nagamitsu, G. Yusakul, T. Miyamoto, H. Tanaka and S. Morimoto, Talanta, 2017, 168, 67-72.

23 Y. Ikeshiro and Y. Tomita, Planta Med., 1985, 51, 390-393.

24 R. W. Jiang, K. L. Wong, Y. M. Chan, H. X. Xu, P. P. But and P. C. Shaw, Phytochemistry, 2005, 66, 2674-2680.

25 K. V. Singh, J. Kaur, G. C. Varshney, M. Raje and C. R. Suri, Bioconjugate Chem., 2004, 15, 168-173. 